\title{
miRNA-22I-3p in Endothelial Progenitor Cell-Derived Exosomes Accelerates Skin Wound Healing in Diabetic Mice
}

This article was published in the following Dove Press journal: Diabetes, Metabolic Syndrome and Obesity: Targets and Therapy

\author{
Juan $\mathrm{Xu}^{1, *}$ \\ Suwen $\mathrm{Bai}^{2, *}$ \\ Yadi Cao' \\ Lei Liu' \\ Yang Fang ${ }^{2}$ \\ Juan $\mathrm{Du}^{2}$ \\ Li Luo' \\ Mingwei Chen' \\ Bing Shen (D) $^{2}$ \\ Qiu Zhang' \\ 'Department of Endocrinology, The First \\ Affiliated Hospital of Anhui Medical \\ University, Hefei, Anhui 230022, People's \\ Republic of China; ${ }^{2}$ School of Basic \\ Medical Sciences, Anhui Medical \\ University, Hefei, Anhui 230032, People's \\ Republic of China \\ *These authors contributed equally to \\ this work
}

Correspondence: Qiu Zhang

Department of Endocrinology, The First Affiliated Hospital of Anhui Medical University, 218 Jixi Road, Hefei, Anhui

230022, People's Republic of China

Tel/Fax +86-55I-6292363I

Email aynfmk@I63.com

Bing Shen

Department of Physiology, School of Basic Medical Sciences, Anhui Medical

University, 8I Meishan Road, Hefei, Anhui

230022, People's Republic of China

Tel +86-55I-65I6II 32

Fax +86-55I-65I6III26

Email shenbing@ahmu.edu.cn
Background: Patients with diabetic cutaneous ulcers experience financial burden and a lower quality of life and life expectancy. Endothelial progenitor cell (EPC)-derived exosomes facilitate skin wound healing by positively modulating vascular endothelial cell function. Exosomes play their important regulatory role through microRNA (miRNA). We explored the potential role and molecular mechanisms of miRNA in EPC-derived exosome healing of diabetic skin wounds.

Methods: Exosomes were isolated from the media of EPCs derived from mice bone marrow. High-throughput sequencing was used to detect the expression of exosome miRNA, and miRNA target genes were predicted using online databases. A diabetic mouse skin wound model was established, and wounds were treated with exosomes, miRNA-221$3 p$, or phosphate-buffered saline.

Results: Exosomes from EPCs accelerated skin wound healing in both control and diabetic mice. High-throughput sequencing showed that miRNA-221-3p was highly expressed in EPCderived exosomes. Skin wound healing in control and diabetic mice was significantly enhanced by EPC-derived exosomes and miRNA-221-3p administration. Immunohistochemical analyses showed that EPC-derived exosomes and miRNA-221-3p increased protein expression levels of the angiogenesis-related factors VEGF, CD31 and cell proliferation marker Ki67. Bioinformatics analyses indicated that miRNA-221-3p may be involved in the AGE-RAGE signaling pathway in diabetic complications, cell cycle, and the p53 signaling pathway.

Conclusion: We concluded that miRNA-221-3p is one of the high-expressed miRNAs in EPC-derived exosomes and promoted skin wound healing in diabetic mice. The finding uncovers the molecular mechanism of EPC-derived exosomes and provides a potential novel approach to the clinical treatment of diabetic skin wounds.

Keywords: endothelial progenitor cells, exosome, diabetes, miRNA-221-3p, wound healing

\section{Introduction}

Diabetes is a group of chronic metabolic diseases affecting more than 340 million people, and approximately $20 \%$ of patients develop diabetic wounds. Leg or foot ulcers are the most common type of wounds in patients with diabetes. ${ }^{1}$ Diabetic cutaneous ulcers are the result of a combination of vascular diseases, neuropathy, and infection. ${ }^{2}$ Among them, vascular diseases can lead to microvascular injuries or microcirculation disorders to varying degrees, which further cause distal ischemia and severe tissue damage. Because of the breakdown of the skin, diabetic cutaneous ulcer is difficult to treat and heal; thus, patients with such ulcers experience financial burdens and have 
a significantly lower quality of life and life expectancy. Therefore, efficient treatments and therapies are urgently needed for this condition.

Endothelial progenitor cells (EPCs) are the precursor cells of vascular endothelial cells, which can be directed to the site of ischemic injury and form new blood vessels through their own differentiation and proliferation, thus improving the ischemic state of limbs. ${ }^{3}$ However, the highsugar environment and inflammatory response of a diabetic wound can lead to a decrease in the number of EPCs, weaken their proliferation, adhesion, and migration to assist in the synthesis of blood vessels, and inhibit the function of blood vessels. ${ }^{4}$ At the same time, advanced glycation end products (AGEs) in the blood vessel wall also reduce EPC activity, thus blocking wound angiogenesis and reducing wound healing. ${ }^{4}$

Exosomes are important carriers of cell autocrine and paracrine information. Exosomes fuse with target cells and transport encapsulated substances, such as proteins, lipids, or RNA, to target cells to mediate the exchange of information between cells and regulate the physiological and pathological functions of target cells. ${ }^{5,6}$ Previous studies have shown that exosomes secreted by EPCs regulate the functions of endothelial cells and play an important role in the repair of vascular endothelial injury. ${ }^{7}$ However, the specific mechanisms underlying those roles remain unclear.

MicroRNA (miRNA) is non-coding small RNA that can inhibit target gene expression by binding to the 3'untranslated region of target mRNA, resulting in either mRNA degradation or inhibition of translation to protein. ${ }^{8}$ Exosomes contain a large number of miRNAs, which have important regulatory functions, including participating in wound healing and angiogenesis. ${ }^{9}$ For example, the expression of miRNA-203 is increased in skin wound tissues, and miRNA-203 modulated wound healing and scar formation via suppressing Hes1 expression in epidermal stem cells. ${ }^{10}$ Exosomes secreted by human adipose-derived mesenchymal stem cells have been shown to transfer miRNA-125a to endothelial cells and to promote angiogenesis by repressing Delta-like 4 (DLL4) expression. ${ }^{11}$ Exosomes have also been found to regulate the function of vascular endothelial cells through miRNAs in studies examining diabetes and various complications. For example, miRNAs, such as miRNA-320a, are enriched in exosomes derived from diabetic cardiomyocytes, leading to enhanced production of vascular endothelial cells. ${ }^{6}$ However, the relationship between miRNAs in EPC-derived exosomes and skin wound healing is still largely unknown.
Thus, the aims of the present study were to characterize the miRNAs in EPC-derived exosomes and determine the effect of endothelial progenitor exosomes on skin wound healing in diabetic mice. We also specifically aimed to clarify the role of miRNA-221-3p in regulating the healing of diabetic skin wounds to determine whether it would be a potential new approach for clinical treatment.

\section{Materials and Methods Culture and Identification of C57BL/6 Mouse EPCs}

This study was approved by the ethics committee of Anhui Medical University. All animal experiments were conducted in accordance with the National Institutes of Health Guide For The Care And Use Of Laboratory Animals (NIH Publication No. 8023, revised 1978). Male C57BL/6 mice were humanely killed by carbon dioxide inhalation. The muscles and connective tissues from the mouse bones were removed under aseptic conditions, and the ends of the tibia and fibula were removed. The bone marrow was extracted with a syringe and then mixed with phosphate-buffered saline (PBS) to form a bone marrow stem cell suspension. The cell suspension was inoculated into a 6-well plate pretreated with human fibronectin, and EGM-2MV medium was added for continuous culture in a $37^{\circ} \mathrm{C}$ incubator. After the cells had adhered, the medium was changed once every 2 days, and the EPCs were obtained after approximately 1 week. Immunofluorescence was used to identify characteristic EPC antigens, as described in Section 2.2.

\section{Immunofluorescence}

Endothelial progenitor cells were incubated on glass slides in the 12-well plate. After the cells attached to the glass, PBS was used to wash the cells for 3 times with an interval of $15 \mathrm{~min}$. Next, the cells were fixed with $4 \%$ paraformaldehyde for $10 \mathrm{~min}$ and washed with PBS for 3 times. Subsequently, $0.2 \%$ Triton $\mathrm{x}-100$ was used for cell permeation and 5\% BSA was used for unspecific antigen blocking at room temperature for $1 \mathrm{~h}$. Then, cells were washed with PBS for 3 times again, and incubated with different primary antibodies (1:50 diluted) at $4^{\circ} \mathrm{C}$ overnight. The next day, the cells were washed with PBS for 3 times, and incubated with Alexa Fluor 488 fluorescence secondary antibody (1:200 diluted, green fluorescence, Invitrogen) for $1 \mathrm{~h}$ in dark at room temperature. Photos were finally taken with a fluorescence microscope. 


\section{Separation and Identification of EPC-Derived Exosomes}

The EPCs were cultured in Dulbecco's Modified Eagle Medium without fetal bovine serum. After $24 \mathrm{~h}$, the medium was collected and centrifuged at $2000 \times g$ for $30 \mathrm{~min}$. The supernatant was added to an exosome separation solution, mixed, and allowed to separate at $4^{\circ} \mathrm{C}$ overnight. Finally, the supernatant was centrifuged at $10,000 \times g$ for $1 \mathrm{~h}$ at $4^{\circ} \mathrm{C}$. The resulting precipitate was the EPC-derived exosomes. ${ }^{12}$ The concentration of the EPC-derived exosomes was determined using a BCA protein concentration assay. Isolated exosomes were stored at $-80^{\circ} \mathrm{C}$ for 1 month.

\section{Transmission Electron Microscopy}

The exosome solution $(10 \mu \mathrm{L})$ was aliquoted, added to a copper grid, and deposited for 2 min. Filter paper was then used to remove any remaining water. Then, $10 \mu \mathrm{L}$ of $2 \%$ uranyl acetate was added to the copper net for $2 \mathrm{~min}$. The retained moisture was waved away using filter paper, and the residual was dried at room temperature for several
A

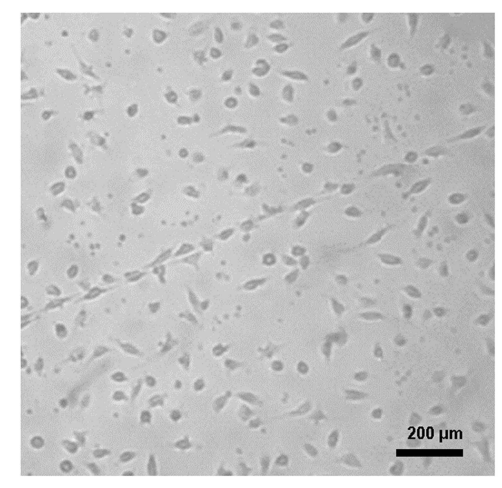

C

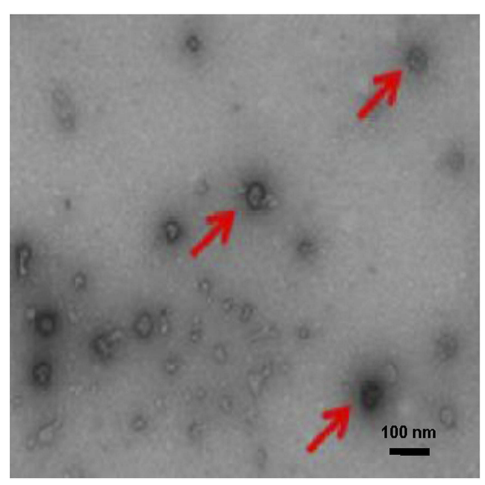

B

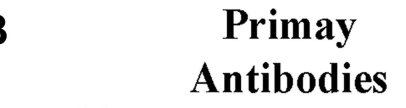

CD133

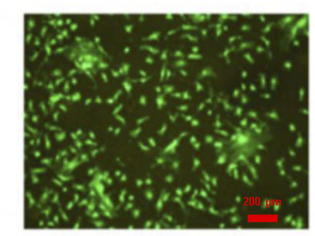

Flk-1

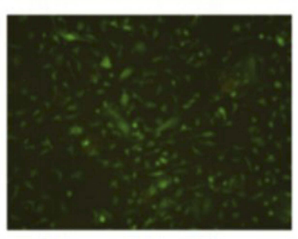

Blank

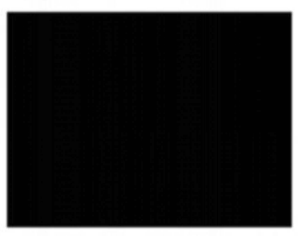

DAPI
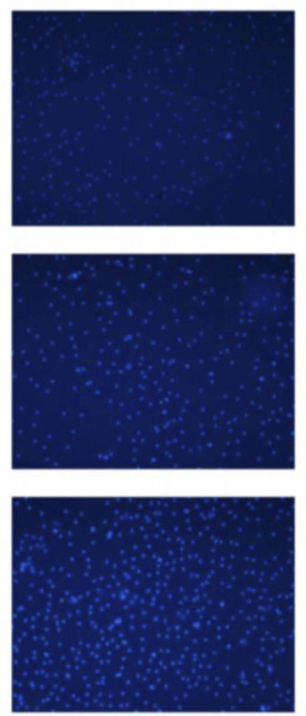

Merge
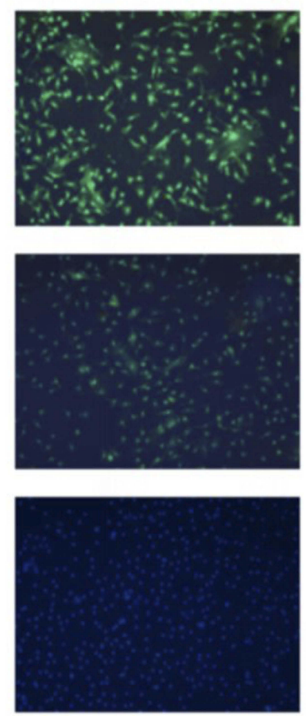

Figure I Identification of EPCs and EPC-derived exosomes. (A) EPC morphology was observed under an optical microscope. (B) Images of the EPC surface-specific proteins CDI33 and Flk-I were captured by immunofluorescence microscopy, with DAPI (4',6-diamidino-2-phenylindole) used to visualize cell nuclei. (C) EPC-derived exosomes were identified by their morphological features under an electron microscope, and the diameters were measured (magnification, 200x). The vesicles shaped like a cupholder are exosomes, as indicated by the red arrows. 
minutes. The exosomes were observed using a transmission electron microscope at a working voltage of $80 \mathrm{kV}$.

\section{Diabetic Mouse Skin Wound Model Establishment and Treatment}

A diabetic mouse model was created using streptozotocin as previously described. ${ }^{13}$ Male C57BL/6 mice (30-35 g) were intraperitoneally injected with streptozotocin (SigmaAldrich, St. Louis, MO, USA) at a dose of $45 \mathrm{mg} / \mathrm{kg}$. After 2 weeks, mice with fasting blood glucose levels higher than $11.1 \mathrm{mmol} / \mathrm{L}$ were selected for further study. Mice were anesthetized with an intraperitoneal injection of $1 \%$ pentobarbital at $40 \mathrm{mg} / \mathrm{kg}$, and their back hair was shaved. Two full-thickness skin excision wounds with a diameter of $6 \mathrm{~mm}$ each were created. The skin wounds were treated with an equal volume of PBS (control group) or EPCderived exosomes $(0.1 \mu \mathrm{g} / \mu \mathrm{L})$ applied directly to the wound twice daily for 12 consecutive days. ImageJ (National Institutes of Health, Bethesda, MD, USA) was used to measure the wound area shown on the images at days $0,3,6,9$, and 12 . Wound healing rate was calculated using the following formula: wound healing rate $(\%)=$ $\left[\left(\mathrm{A}_{0}-\mathrm{A}_{\mathrm{t}}\right) / \mathrm{A}_{0}\right] \times 100 \%$, where $\mathrm{A}_{0}$ is the initial wound area and $A_{t}$ is the wound area on day 3,6, 9 or 12 . The miRNA-221-3p was diluted to $0.5 \mu \mathrm{mol} / \mathrm{L}$ using OPTIMEM and then used to treat skin wounds in normal and diabetic mice.

\section{Immunohistochemical Analysis}

Skin wound specimens from normal and diabetic mice were fixed with $4 \%$ paraformaldehyde and embedded in paraffin as previously described by our group. ${ }^{14}$ The specimens were cut into 5- $\mu \mathrm{m}$-thick sections, dewaxed, and blocked. The endogenous peroxidases were inactivated with $0.3 \%$ hydrogen peroxide $\left(\mathrm{H}_{2} \mathrm{O}_{2}\right)$. The non-specific binding sites in the tissues were blocked with normal goat serum and allowed to stand at room temperature for $30 \mathrm{~min}$. Then, the primary antibody (1:50) was added and incubated overnight at $4{ }^{\circ} \mathrm{C}$. The next day, the horseradish peroxidase-labeled secondary antibody (1:100) was incubated for $1 \mathrm{~h}$, and the $\mathrm{ABC}$ complex was added dropwise and incubated at room temperature for $1 \mathrm{~h}$. Finally, 3'-diaminobenzidine- $\mathrm{H}_{2} \mathrm{O}_{2}$ was used for color development, and hematoxylin was used to stain cell nuclei. Images of stained sections were captured using a light microscope and analyzed with Image Pro Plus, version 5.1 (Media Cybernetics, Rockville, MD, USA), software.

\section{miRNA Analysis, Target Gene Prediction,} and Functional Enrichment

The miRNA sequencing was performed on the extracted EPC exosomes. FastQC (version 0.11.5) software was used for quality inspection of the original data. Sequencing reads that passed quality control were mapped to the mouse reference genome (MMU10) available from Ensembl genome browser 94 using the miRDeep2 (version 0.0.7) package. The mapper.pl module of the miRDeep2

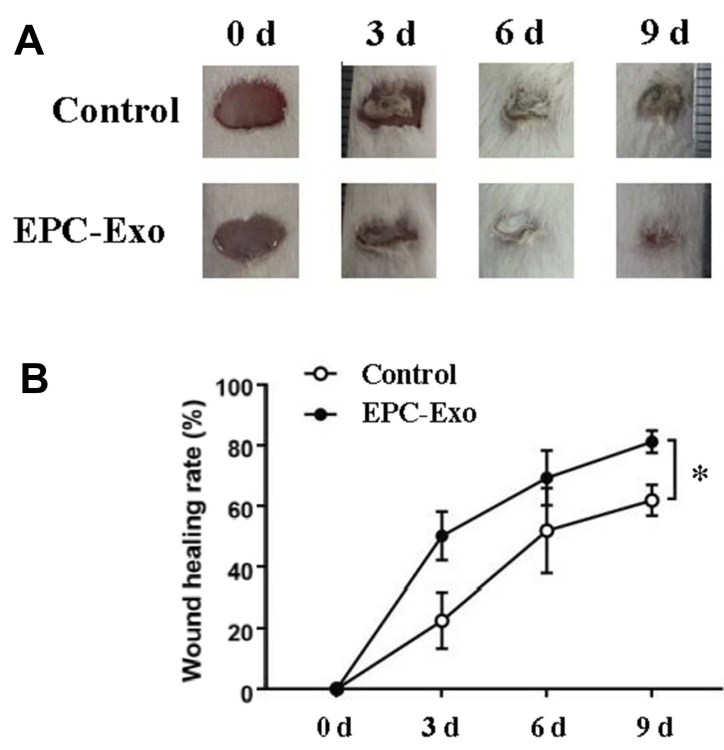

C

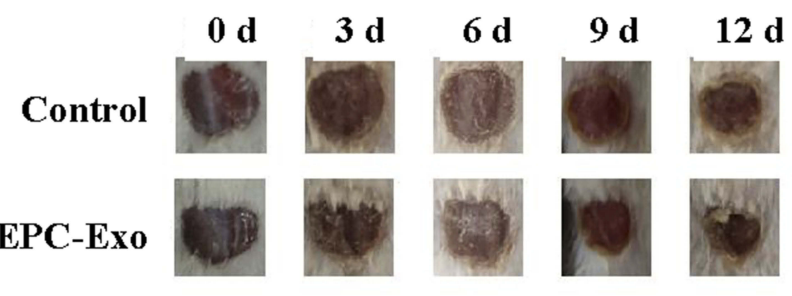

D

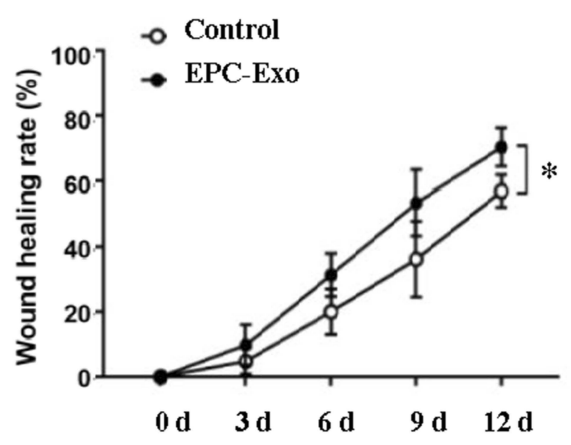

Figure 2 EPC-derived exosomes promote skin wound healing in normal and diabetic mice. Wound area changes $(\mathbf{A})$ and wound healing rate $(\mathbf{B})$ with treatment of EPC-derived exosomes (EPC-Exo) or with PBS (control) for 9 days in normal mice. Values represent the mean $\pm S E(n=3) ; * P<0.05$. Wound area changes (C) and wound healing rate (D) with treatment of EPC-derived exosomes or with PBS for 12 days in diabetic mice. Values represent the mean $\pm S E(n=5)$; $* P<0.05$. 
tool was run with the following parameters: $-c-j-m-q-p$ $-s-t-v$. This process generated non-redundant readings using a count of mapped readings, source, and genome locations. The mature and hairpin miRNA sequences were obtained from miRBase release 18 (http://www.mirbase. org). Finally, the resulting FASTQ files were processed by miRDeep2.pl script, and the read count was extracted for each miRNA. The miRNA target genes were predicted online using RNA22-HAS (https://cm.jefferson.edu/ rna22/), miRDB (http://mirdb.org/), and TargetScan (http://www.targetscan.org/mamm_31/), and the intersection of the three results was taken. Kyoto Encyclopedia of Genes and Genomes (KEGG) enrichment analysis was conducted online using Metascape (http://metascape.org) to predict the signaling pathways in which miRNA may participate. $^{15}$

\section{Statistical Analysis}

All statistical analyses were performed using GraphPad Prism software (version 7.04). Unless otherwise stated, data are expressed as the mean \pm standard error (SE). The MannWhitney test (two-tailed) and two-way analysis of variance (ANOVA) were used to compare the results between groups. $P$ values $<0.05$ were considered statistically significant.

\section{Results}

\section{Identification of EPCs and EPC-Derived Exosomes}

We separated EPCs by inducing differentiation of mouse bone marrow monocytes and detecting the expression of the surfacespecific proteins CD133 and Flk-1 on EPCs by using immunofluorescence. The results showed that the EPCs were typically
A

CD31
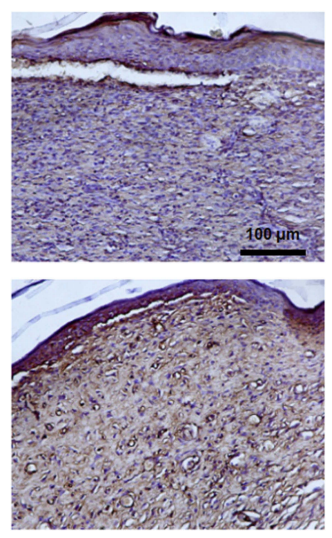

B

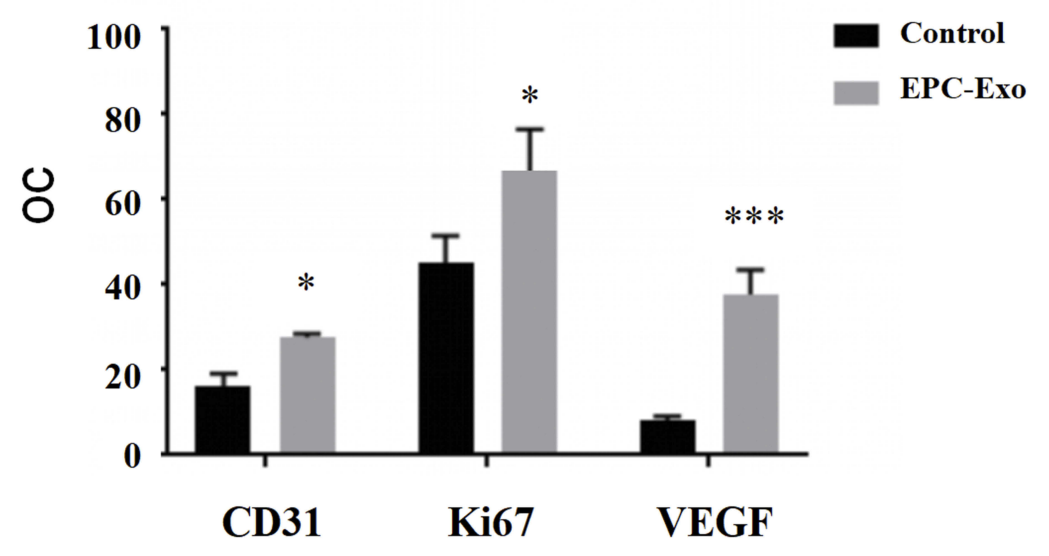

Figure 3 Immunohistochemical staining of skin wound tissue treated with EPC-derived exosomes in normal mice. Representative immunohistochemical images (A) and summary data (B) of immunostaining in skin wound tissue obtained from normal mice treated with EPC-derived exosomes or PBS (control), expressed as integrated optical density/total area (OC) (magnification, 200x). All values are presented as means and standard deviations $(n=3)$. Statistically significant differences are indicated by $* P<0.05$, $* * * \mathrm{P}<0.001$, compared with the control group. 
linearly distributed (Figure 1A) and that both CD133 and Flk-1 were strongly expressed (Figure 1B). ${ }^{5}$ EPC-derived exosomes were identified by their morphological features under an electron microscope, and the diameter of the exosomes was measured. As shown in Figure 1C, exosomes were typically "cupholder"-shaped, with diameters ranging from 40 to 100 $\mathrm{nm}$, indicating successful isolation of EPC-derived exosomes.

\section{EPC-Derived Exosomes Promote Wound Healing in Diabetic Mice}

We applied isolated EPC-derived exosomes $(0.1 \mu \mathrm{g} / \mu \mathrm{L})$ or PBS (as a control) directly to skin wounds of normal and diabetic mice. As shown in Figure 2, both normal mice and diabetic mice treated with EPC-derived exosomes showed accelerated wound closure compared with that in PBS-treated mice. In addition, we used immunohistochemical analysis to evaluate the expression levels of the angiogenesis-related factors VEGF, CD31 and cell proliferation marker Ki67 in mouse skin tissue. The results showed that the expression levels of VEGF, CD31, and Ki67 were significantly increased in the skin tissue treated with the EPC-derived exosomes compared with that following treatment of PBS (the control group) in both normal mice (Figure 3) and diabetic mice (Figure 4), suggesting that EPC-derived exosomes may accelerate wound healing by promoting angiogenesis.

\section{High-Throughput Sequencing of miRNAs in EPC-Derived Exosomes}

EPC-derived exosomes contain multiple regulatory substances, including miRNAs, which have been shown to be involved in angiogenesis. To determine whether miRNAs in EPC-derived exosomes play an important role in wound healing, we used high-throughput sequencing to characterize the miRNA abundance. As shown in Figure 5A, all the base position masses were above a quality score of 30 , which indicated that we obtained high-quality reads that could be used for further analyses. R language was used to

A

CD31

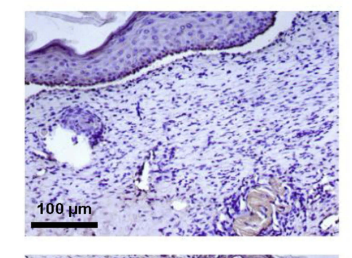

Control

EPC-Exo

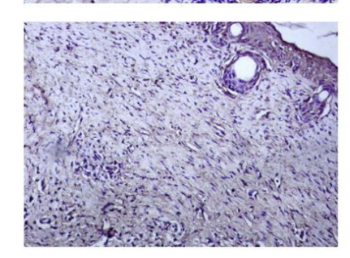

Ki67

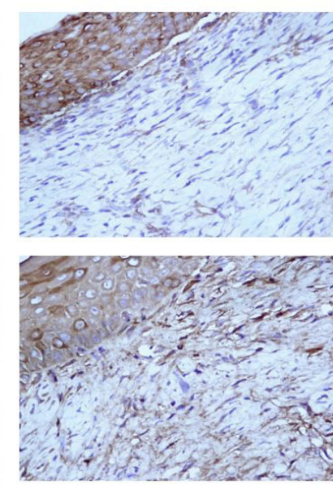

VEGF

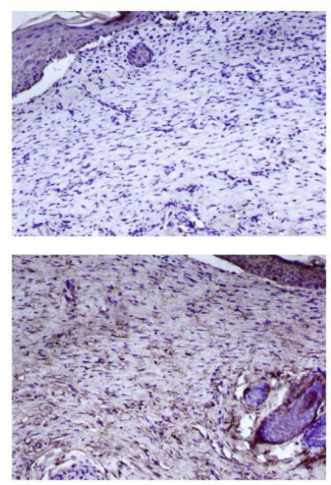

Control

EPC-Exo

B

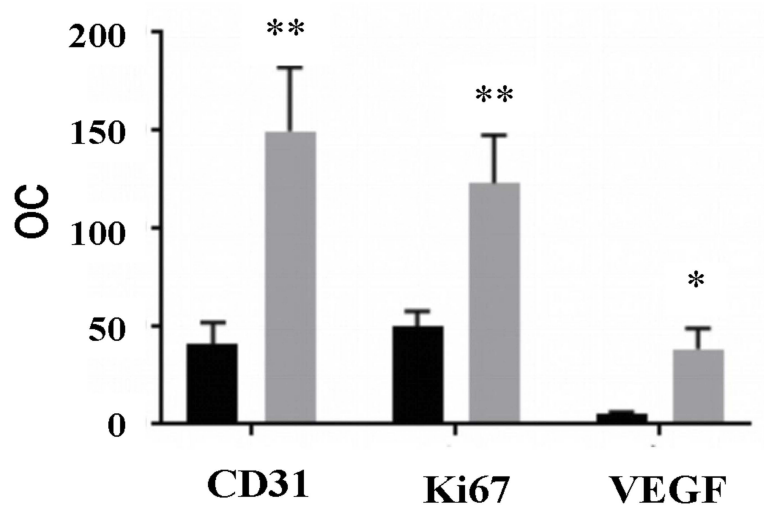

Figure $4 \mathrm{Immunohistochemical} \mathrm{staining} \mathrm{of} \mathrm{skin} \mathrm{wound} \mathrm{tissue} \mathrm{treated} \mathrm{with} \mathrm{EPC-derived} \mathrm{exosomes} \mathrm{in} \mathrm{diabetic} \mathrm{mice.} \mathrm{Representative} \mathrm{immunohistochemical} \mathrm{images} \mathrm{(A)} \mathrm{and}$ summary data (B) of immunostaining in skin wound tissue obtained from diabetic mice treated with EPC-derived exosomes or PBS (control), expressed as integrated optical density/total area (OC) (magnification, 200x). All values are presented as means and standard deviations $(n=3)$. Statistically significant differences are indicated by $* P<0.05$ and $* * \mathrm{P}<0.01$, compared with the control group. 
A

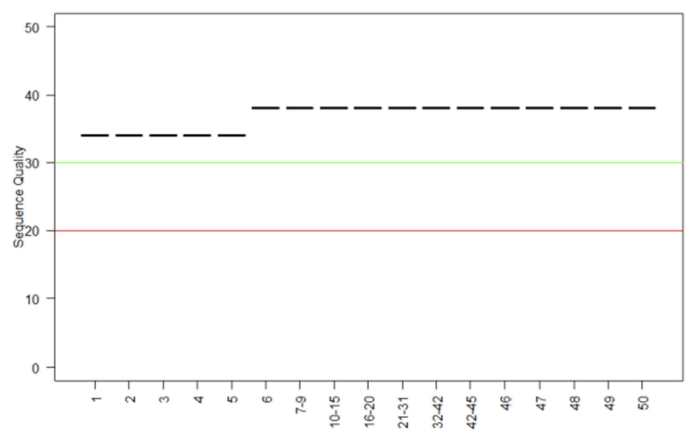

B

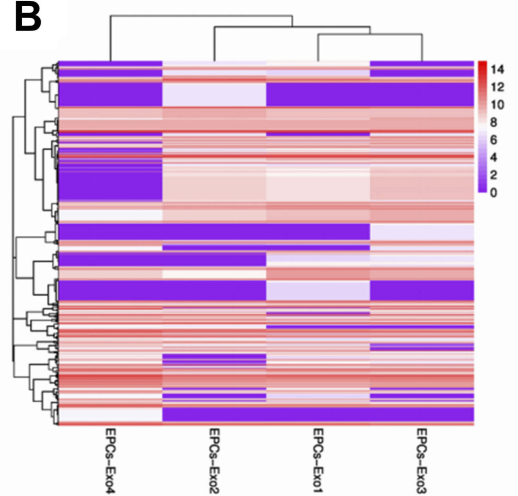

C

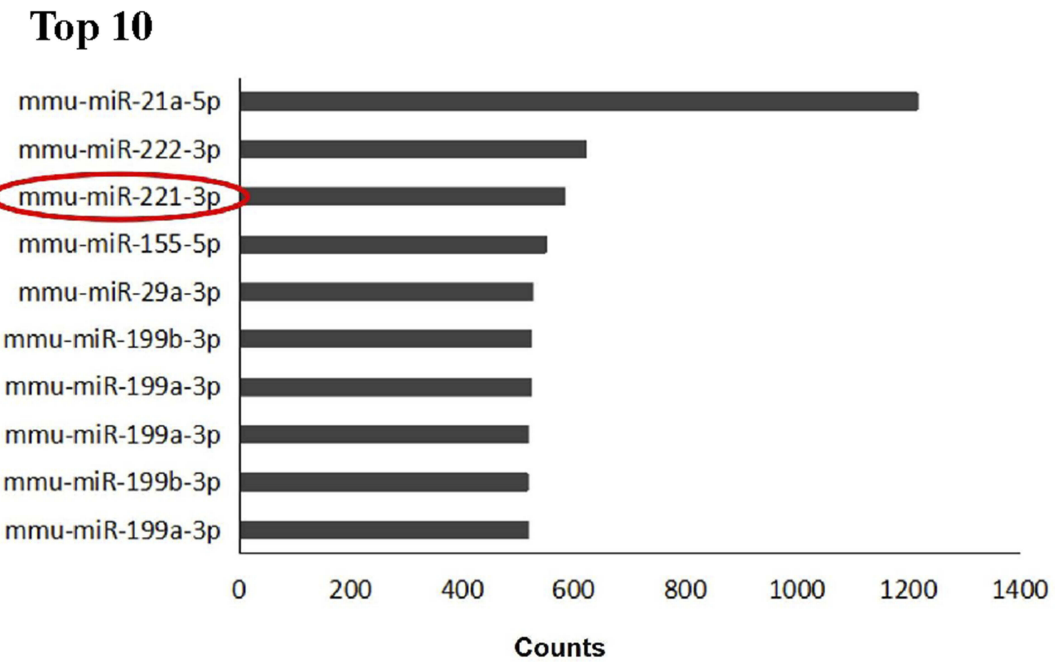

Figure 5 High-throughput sequencing of miRNAs in EPC-derived exosomes. (A) Sequencing quality. A block diagram showing the quality of four sample read sequences. The $\mathrm{X}$-axis is the base position of each reading. The $\mathrm{Y}$-axis is the sequence quality score. A median value of the sequencing quality score less than 20 indicates poor sequencing quality, and a value higher than 30 indicates better sequencing quality. (B) Cluster analysis of miRNA expression profiles. Red represents high expression, purple represents low expression. (C) The top 10 miRNAs expressed in endothelial progenitor exosomes. MiRNA-22I-3p was indicated by red circle.

cluster the miRNA expression profile, and the results showed that the miRNA expression pattern in the endothelial EPC group was similar (Figure 5B). In addition, we sequenced the expression levels of the EPC exosome miRNAs and listed the top 10 miRNAs of those totally expressed. The results showed that miRNA-21a$5 p$, miRNA-222-3p, and miRNA-221-3p were highly expressed in EPC-derived exosomes (Figure 5C).

\section{miRNA-22I-3p Promotes Wound Healing in Diabetic Mice}

We used the top three miRNAs expressed in EPC exosomes to treat normal and diabetic mouse skin wounds. Although miRNA-21a-5p (Supplementary Figure 1A) and
miRNA-222-3p (Supplementary Figure 1B) had no significant effect on skin wound healing in mice, when miRNA221-3p at a dose of $0.5 \mu \mathrm{mol} / \mathrm{L}$ was applied directly to the wounds of normal or diabetic mice, the healing rates of both normal and diabetic mice were significantly increased and wound scab formation occurred sooner (Figure 6). The results of immunohistochemistry showed that the expression levels of the angiogenesis-related factors VEGF, CD31 and cell proliferation marker Ki67 were also significantly increased in the miRNA-221-3p-treated groups of normal (Figure 7) and diabetic (Figure 8) mice. These data indicated that miRNA-221-3p promotes wound healing in mice and contributes to the regulation of angiogenesis by EPC-derived exosomes. 


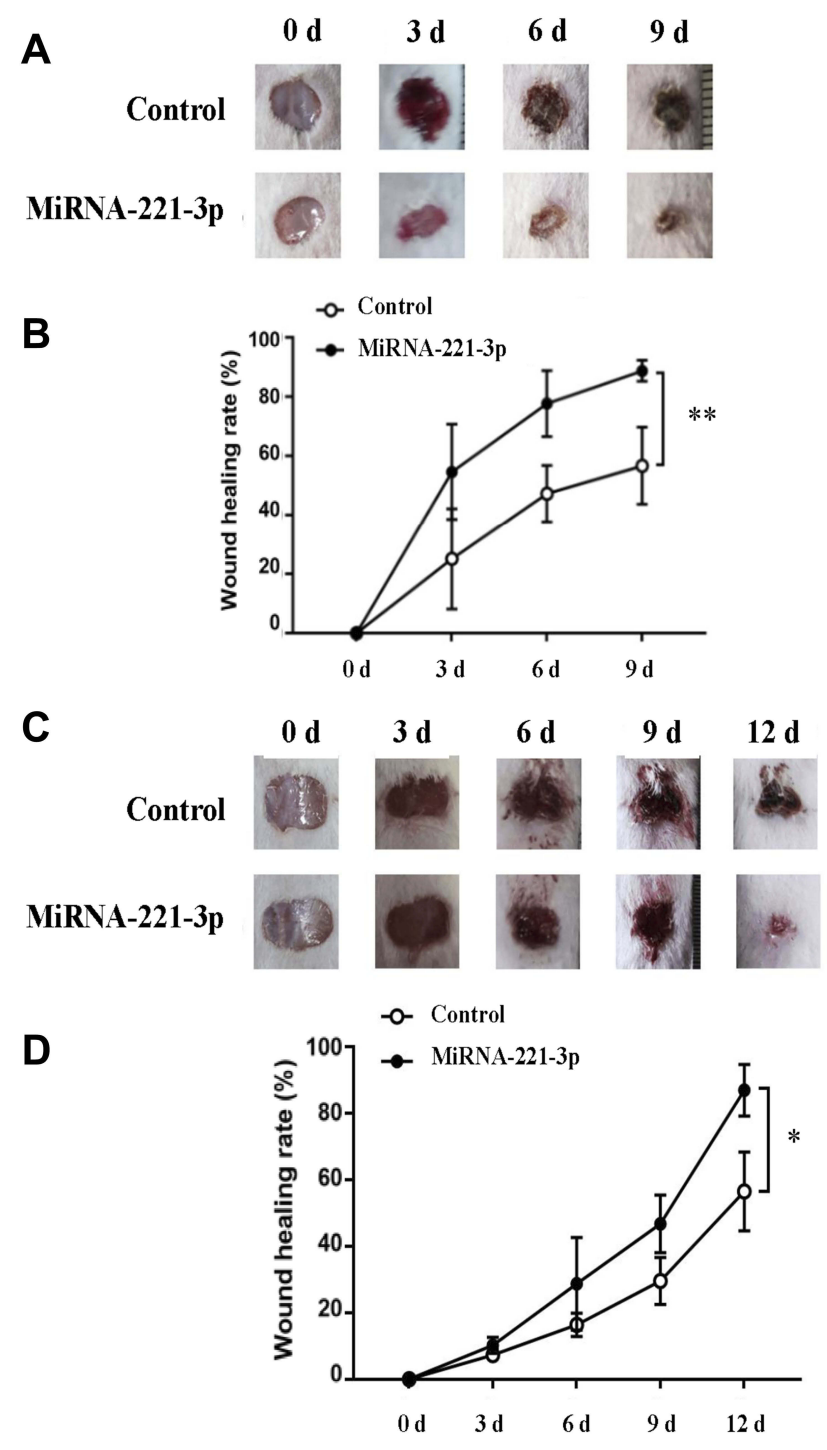

Figure 6 MiRNA-22I-3p promotes skin wound healing in normal and diabetic mice. Skin wound area changes (A) and wound healing rate (B) in normal mice treated with miRNA-22I-3p $(0.5 \mu \mathrm{mol} / \mathrm{L})$ or OPTI-MEM (control) for 9 days. Values represent the mean $\pm S E(n=5) ; * * P<0.01$. Skin wound area changes $(\mathbf{C})$ and wound healing rate (D) in diabetic mice treated with miRNA-22I-3p or OPTI-MEM for 12 days. Values represent the mean \pm SE $(n=5)$; $* P<0.05$.

\section{Target Gene Prediction and KEGG Pathway Analysis of miRNA-22I-3p}

We predicted the target genes of miRNA-221-3p by using bioinformatic tools, and we performed a KEGG analysis to predict the potential signaling pathways related to miRNA-221-3p. The results showed that the target genes of miRNA-221-3p may be involved in the AGERAGE signaling pathway in diabetic complications, cell cycle, p53-signaling pathway, and MAPK signaling pathway, among others (Figure 9A). These results indicated that miRNA-221-3p has a potential to downregulate the expression levels of p27, caspase-3, E-selectin, c-Jun
N-terminal kinase, and other proteins involved in the AGE-RAGE signaling pathway. Our analysis suggested that miRNA-221-3p likely antagonizes AGE-induced cell hypertrophy, apoptosis, inflammatory response, and vascular dysfunction (Supplementary Figure 2A). In addition, miRNA-221-3p might also downregulate the expression of the cyclin-dependent kinase inhibitors p27 and p57 in the cell cycle signaling pathway (Supplementary Figure 2B), which could promote cell proliferation as a result. These data suggested that miRNA-221-3p may play an important role in the regulatory function of EPCderived exosomes.

\section{Discussion}

Diabetic cutaneous ulcer (DCU), a common chronic complication of diabetes, causes severe disability. ${ }^{16,17}$ The specific mechanism of difficult healing of diabetic wounds is still not fully understood. In recent years, research has found that it may be related to the special microenvironment of the skin of diabetic patients, the reduction of angiogenesis, the change in the number of related inflammatory factors and the dysfunction of immune response. ${ }^{2}$ Among them, microvascular and microcirculatory disorders of different degrees caused by vascular diseases are the main causes of distal ischemia and tissue damage. ${ }^{18}$ Studies have shown that under the influence of high-glucose environment and ROS in vivo, inflammatory cells such as macrophages can continuously generate a large number of inflammatory precursor factors, maintain the occurrence of inflammatory reactions, inhibit wound healing, and inhibit the formation and function of new blood vessels. ${ }^{4,19}$ Long-term chronic inflammatory response can also inhibit angiogenesis by down-regulating the expression of vascular endothelial growth factor (VEGF) and angiogenin-1 (ANG1) and their receptors, inhibiting their ability to promote the proliferation and adhesion of EPCs. ${ }^{20}$

Restoration of blood flow to injured tissue facilitates the oxygen and nutrient supply to support the growth of reparative cells that promote wound healing. ${ }^{21,22}$ Previous studies have shown that EPC-derived exosomes (EPCExos) can regulate the function of endothelial cells and play an important role in the repair of injured vascular endothelium. ${ }^{23-26} \mathrm{Li}$ et al demonstrated in animal experiments that EPC-Exos can accelerate the early reendothelialization after carotid artery endothelial injury in rats, thus promoting vascular repair. In vitro experiments, endothelial progenitor cell exosomes were also found to enhance the proliferation and migration of endothelial 


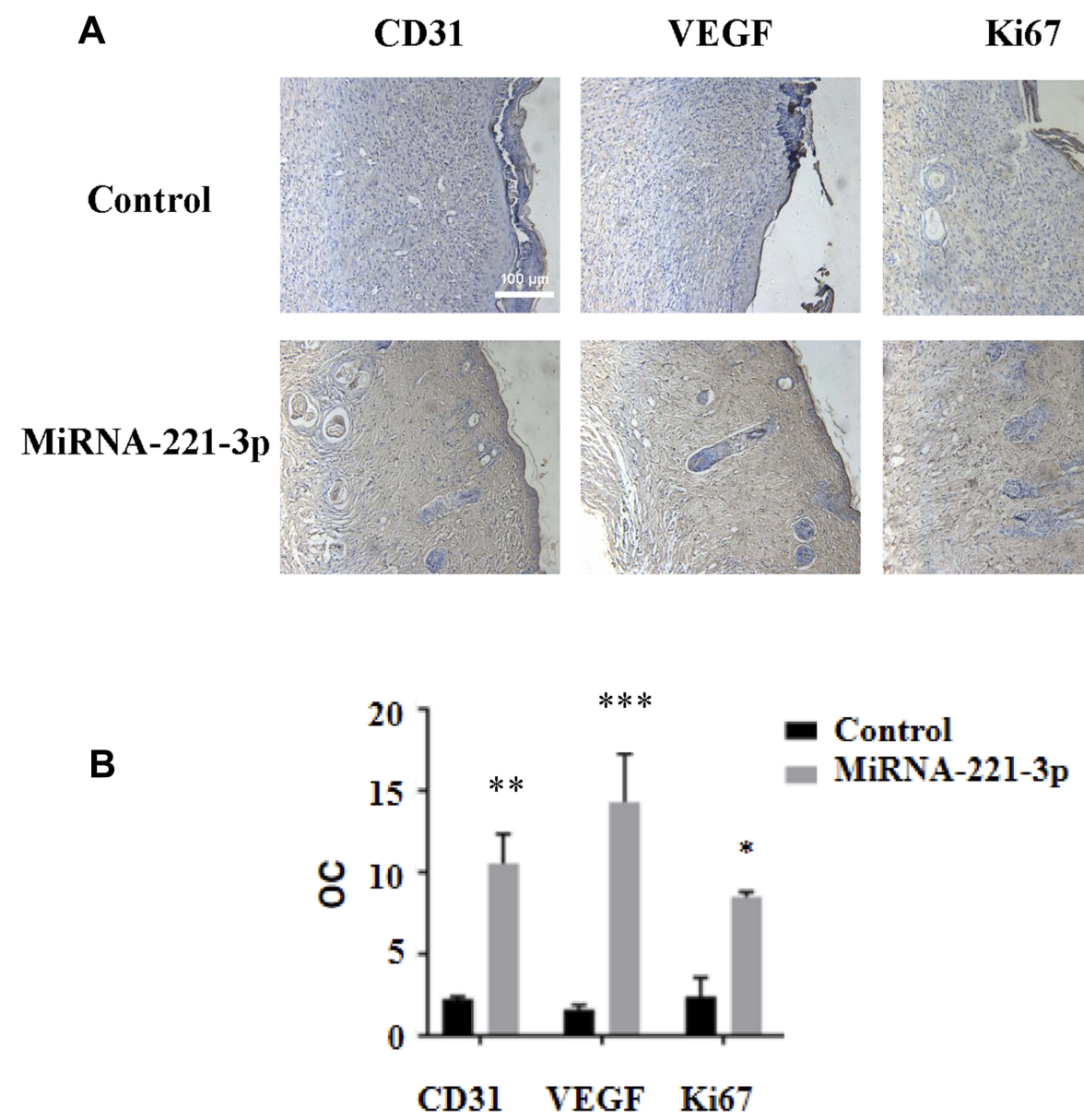

Figure 7 Immunohistochemical staining of miRNA-22I-3p-treated skin wound tissue in normal mice. Representative immunohistochemical images (A) and summary data (B) showing immunostaining of the skin wound tissue from normal mice treated with miRNA-22I-3p (expressed as integrated optical density/total area; OC) (magnification, $200 \times)$. All values are presented as means and standard deviations $(n=3)$. Statistically significant differences are indicated by $* P<0.05$, $* * P<0.0 \mathrm{I}$, $* * * P<0.00 \mathrm{I}$, compared with the control group treated with PBS.

cells and increase the expression of angiogenic-related molecules. ${ }^{5}$ Zhang et al showed that EPC-Exos can stimulate the angiogenic activity of endothelial cells by activating Erk1/2 signal transduction, and ultimately promote skin wound repair and regeneration. ${ }^{27}$ In the current investigation, we found that exosomes derived from endothelial progenitor cells promoted skin wound healing and expression of angiogenesis-related molecules in diabetic mice, which is consistent with the report.

It has been reported in the studies on diabetes and its complications that exosomes can regulate vascular endothelial cells through miRNA. ${ }^{28,29}$ For example, miRNA-221 targets and binds to significant genes and key factors in the insulin/insulin-like growth factor-1 signaling pathway, and has been previously shown to be related to metabolic syndrome as well as to diabetes and its complications. ${ }^{30}$ Previous studies have shown that miRNA-221 expression is increased in the high-glucose state, while c-kit expression is decreased. The miRNA221-c-kit pathway may damage the migration of endothelial cells, leading to vascular dysfunction in patients with diabetes. ${ }^{31}$ A recent clinical study showed that miRNA221 was significantly increased as the severity of diabetic retinopathy increased, suggesting that miRNA-221 could be used as a potential biomarker for diabetic retinopathy. ${ }^{32}$ However, no studies have, to our knowledge, investigated the role of miRNA-221-3p in skin wound healing.

In this study, we explored an emerging role of miRNA221-3p in the healing of diabetic skin wounds by using transcriptomics, animal experiments, and bioinformatics analyses. Our data showed, for the first time, that treatment with miRNA-221-3p accelerated skin wound healing in both normal and diabetic mice. VEGF is one of the most potent known angiogenic cytokines in the skin, and the amount of VEGF present in a wound affects healing and supports the rate-limiting steps of vasculogenesis and angiogenesis. ${ }^{1,33}$ 
A

CD31

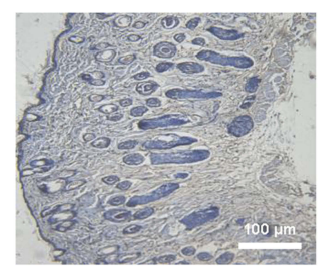

Control

MiRNA-221-3p

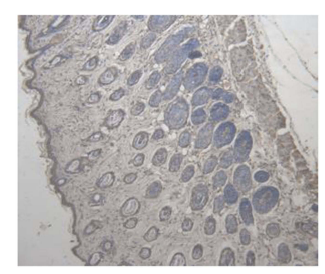

VEGF
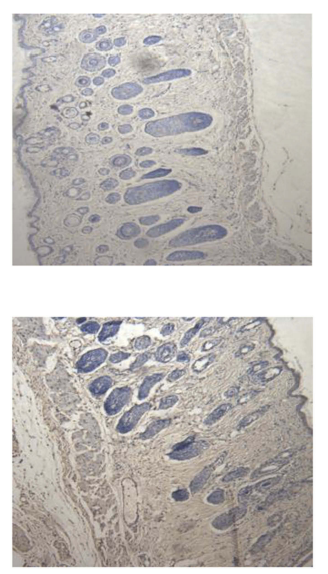

Ki67
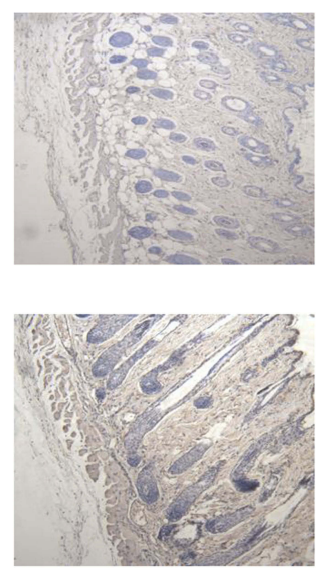

B

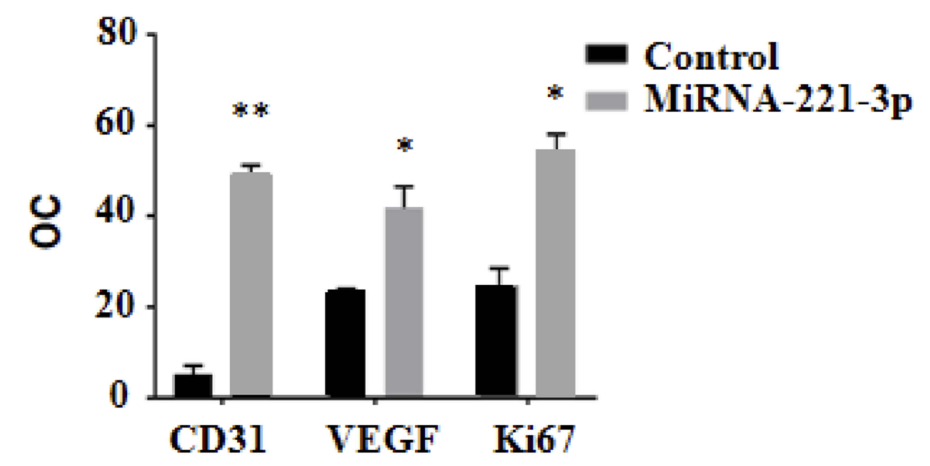

Figure 8 Immunohistochemical staining of miRNA-22I-3p-treated skin wound tissue in diabetic mice. Representative immunohistochemical images (A) and summary data (B) showing immunostaining in the skin wound tissue from diabetic mice treated with miRNA-22I-3p (expressed as integrated optical density/total area; OC) (magnification, 200x). All values are presented as means and standard deviations $(n=3)$. Statistically significant differences are indicated by $* P<0.05$ and $* * P<0.01$, compared with the control group treated with PBS.

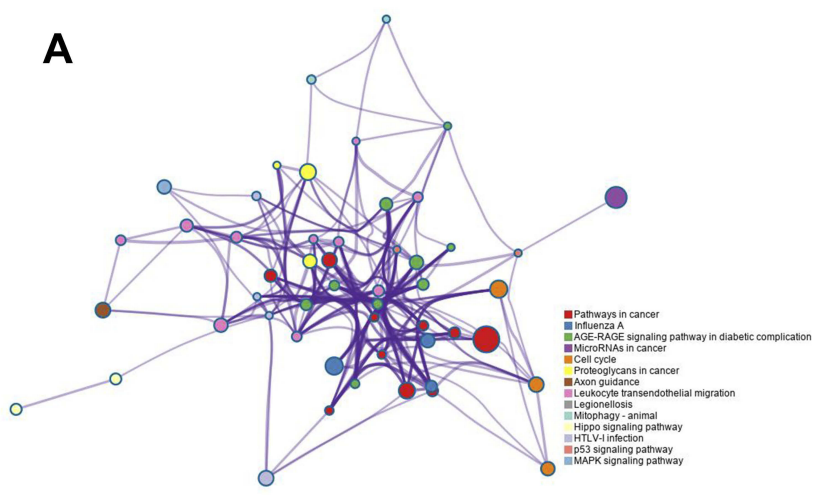

Figure 9 Target gene prediction and KEGG signal analysis of miRNA-22I-3p. The miRNA-22I-3p target genes were predicted online using RNA22-HAS, miRDB, and TargetScan. The KEGG enrichment analysis was conducted online using Metascape to predict the signaling pathways in which miRNA-22I-3p may participate.

Our immunohistochemical results suggested that miRNA221-3p increased the protein expression levels of VEGF, further suggesting that miRNA-221-3p can promote angiogenesis and thereby improve blood perfusion and the metabolism of injured tissue. The presence of Ki67 is a good indicator of cell proliferation, and it plays an important role in the initiation of cell proliferation. ${ }^{34}$ Our results also showed that miRNA-221-3p increased the expression levels of Ki67 in the skin tissue of diabetic mice. Taken together, these data suggest that angiogenesis and cell proliferation are enhanced in miRNA-221-3p-treated mice.

AGEs and their receptors (RAGE) are involved in and mediate various signaling pathways of oxidative stress, inducing the generation of reactive oxygen species and activating nuclear factor-kappa B (NF-kB), thereby leading to inflammatory reactions, cell apoptosis, and microvascular diseases. $^{35}$ Therefore, it is an important strategy for the prevention and treatment of type 2 diabetes mellitus and its complications to effectively inhibit the AGE-RAGE pathway and reduce the reaction of the NF-kB pathway, thus 
contributing to the reduction of oxidative stress injury. ${ }^{36}$ Our KEGG analyses showed that miRNA-221-3p may downregulate the expression levels of key proteins in the AGERAGE signaling pathway. In addition, our results showed that miRNA-221-3p could downregulate the expression of p27 and p57 proteins in the cell cycle signaling pathway. Cyclin-dependent kinase inhibitors are negative regulatory factors during cell cycle progression. ${ }^{37}$ Therefore, we speculate that miRNA-221-3p promotes the proliferation of vascular cells by inhibiting the negative regulators of the cell cycle. However, this speculation and the specific mechanism of miRNA-221-3p will require further study.

Our results may have potential clinical implications. Our study showed that EPC-derived exosomes expressed a high level of miRNA-221-3p and that treating skin wounds of mice with EPC-derived exosomes had an effect similar to that observed after administration of miRNA-221-3p. However, treatment with EPC-derived exosomes is more feasible and less difficult in clinical practice than the administration of miRNA, which would require the use of transfection reagents. Different routes of exosome administration have been described, such as systematic administration, ${ }^{38}$ intranasal administration, ${ }^{39}$ or subcutaneous injection. ${ }^{25}$ Although applying exosomes by directly spreading them onto wound sites has been seldomly described, it was the most convenient and practical method for use in the mouse model in the present study. Thus, additional studies are warranted to determine whether this route of administration would be effective in clinical practice, which would expand exosome-based therapeutics to a large extent.

Despite the mechanistic strength, we admit that our study has some limitations that should be addressed in the future. First, our immunofluorescence experiments in relation to exosomal markers can be substantiated by qPCR experiments. Similarly, due to some constraints, we could not study alterations in transcriptional signatures of cytokines and growth factors in relation to wound healing as these could be direct or indirect targets of miRNA-221-3p. As our KEGG analyses showed that miRNA-221-3p may downregulate the expression levels of key proteins in the AGE-RAGE signaling pathway, we also emphasize that future studies are warranted to demonstrate the extent of transcriptional levels of certain AGE-RAGE signatures in the wound healing model.

\section{Conclusions}

In conclusion, we demonstrated that miRNA-221-3p is highly expressed in EPC-derived exosomes, and promoted skin wound healing in normal and diabetic mice. This finding reveals the molecular mechanism of EPC-derived exosomes and provides a potential novel target and method for clinical treatment of diabetic skin wounds.

\section{Acknowledgments}

The current work was supported by grants from the National Natural Science Foundation of China (grant Nos. 81970703, 81570403 and U1732157). Juan Xu and Suwen Bai are co-first authors for this study.

\section{Author Contributions}

J.X., S.B., Y.C. and Y.F. performed experiments and analyzed data; J.X., L.L., B.S. and Q.Z. wrote the manuscript; J. D., B.S., L.L, M.C and Q.Z. supervised the project. All authors contributed to data analysis, drafting or revising the article, gave final approval of the version to be published, and agree to be accountable for all aspects of the work.

\section{Disclosure}

All authors report no conflicts of interest in this work.

\section{References}

1. Patel S, Srivastava S, Singh MR, et al. Mechanistic insight into diabetic wounds: pathogenesis, molecular targets and treatment strategies to pace wound healing $[\mathrm{J}]$. Biomed Pharmacother. 2019;112:108615. doi:10.1016/j.biopha.2019.108615

2. Paschou SA, Stamou M, Vuagnat H, et al. Pain management of chronic wounds: diabetic ulcers and beyond[J]. Maturitas. 2018;117:17-21. doi:10.1016/j.maturitas.2018.08.013

3. Balaji S, King A, Crombleholme TM, et al. The role of endothelial progenitor cells in postnatal vasculogenesis: implications for therapeutic neovascularization and wound healing[J]. Adv Wound Care. 2013;2(6):283-295. doi:10.1089/wound.2012.0398

4. Drela E, Stankowska K, Kulwas A, et al. Endothelial progenitor cells in diabetic foot syndrome[J]. Adv Clin Exp Med. 2012;21 (2):249-254.

5. Li X, Chen C, Wei L, et al. Exosomes derived from endothelial progenitor cells attenuate vascular repair and accelerate reendothelialization by enhancing endothelial function[J]. Cytotherapy. 2016;18 (2):253-262. doi:10.1016/j.jcyt.2015.11.009

6. Beuzelin D, Kaeffer B. Exosomes and miRNA-Loaded biomimetic nanovehicles, a focus on their potentials preventing type-2 diabetes linked to metabolic syndrome[J]. Front Immunol. 2018;9:2711. doi:10.3389/fimmu.2018.02711

7. Li X, Jiang C, Zhao J. Human endothelial progenitor cells-derived exosomes accelerate cutaneous wound healing in diabetic rats by promoting endothelial function[J]. $J$ Diabetes Complications. 2016;30(6):986-992. doi:10.1016/j.jdiacomp.2016.05.009

8. Qin B, Cao Y, Yang H, et al. MicroRNA-221/222 regulate ox-LDLinduced endothelial apoptosis via Ets-1/p21 inhibition[J]. Mol Cell Biochem. 2015;405(1-2):115-124. doi:10.1007/s11010-015-2403-5

9. Li Y, Shen Z, Yu XY. Transport of microRNAs via exosomes[J]. Nat Rev Cardiol. 2015;12(4):198. doi:10.1038/nrcardio.2014.207-c1

10. Zhou Z, Shu B, Xu Y, et al. microRNA-203 modulates wound healing and scar formation via suppressing hes1 expression in epidermal stem cells[J]. Cell Physiol Biochem. 2018;49(6):2333-2347. doi:10.1159/ 000493834 
11. Liang X, Zhang L, Wang S, et al. Exosomes secreted by mesenchymal stem cells promote endothelial cell angiogenesis by transferring miR-125a[J]. J Cell Sci. 2016;129(11):2182-2189. doi:10.1242/ jcs. 170373

12. Wang C, Chen L, Huang Y, et al. Exosome-delivered TRPP2 siRNA inhibits epithelial-mesenchymal transition of $\mathrm{FaDu}$ cells[J]. Oncol Lett. 2019;17(2):1953-1961. doi:10.3892/o1.2018.9752

13. Ye CL, Shen B, Ren XD, et al. An increase in opening of $\mathrm{BK}(\mathrm{Ca})$ channels in smooth muscle cells in streptozotocin-induced diabetic mice[J]. Acta Pharmacol Sin. 2004;25(6):744-750.

14. Wu K, Shen B, Jiang F, et al. TRPP2 enhances metastasis by regulating epithelial-mesenchymal transition in laryngeal squamous cell carcinoma[J]. Cell Physiol Biochem. 2016;39(6):2203-2215. doi:10.1159/000447914

15. Fang Y, Wang P, Xia L, et al. Aberrantly hydroxymethylated differentially expressed genes and the associated protein pathways in osteoarthritis[J]. PeerJ. 2019;7:e6425. doi:10.7717/peerj.6425

16. Brem H, Tomic-Canic M. Cellular and molecular basis of wound healing in diabetes[J]. J Clin Invest. 2007;117(5):1219-1222. doi:10.1172/JCI32169

17. Bus SA, Waaijman R, Arts M, et al. Effect of custom-made footwear on foot ulcer recurrence in diabetes: a multicenter randomized controlled trial[J]. Diabetes Care. 2013;36(12):4109-4116. doi:10.2337/ dc13-0996

18. Alavi A, Sibbald RG, Mayer D, et al. Diabetic foot ulcers: part I. Pathophysiology and prevention[J]. J Am Acad Dermatol. 2014;70(1):19-20. doi:10.1016/j.jaad.2013.06.055

19. Menghini R, Uccioli L, Vainieri E, et al. Expression of tissue inhibitor of metalloprotease 3 is reduced in ischemic but not neuropathic ulcers from patients with type 2 diabetes mellitus[J]. Acta Diabetol. 2013;50(6):907-910. doi:10.1007/s00592-013-0478-6

20. Losi P, Briganti E, Errico C, et al. Fibrin-based scaffold incorporating VEGF- and bFGF-loaded nanoparticles stimulates wound healing in diabetic mice[J]. Acta Biomater. 2013;9(8):7814-7821. doi:10.1016/j. actbio.2013.04.019

21. Carmeliet P. Angiogenesis in health and disease[J]. Nat Med. 2003;9 (6):653-660. doi:10.1038/nm0603-653

22. Broughton GN, Janis JE, Attinger CE. The basic science of wound healing[J]. Plast Reconstr Surg. 2006;117(7Suppl):12S-34S. doi:10. 1097/01.prs.0000225430.42531.c2

23. Kim JY, Song SH, Kim KL, et al. Human cord blood-derived endothelial progenitor cells and their conditioned media exhibit therapeutic equivalence for diabetic wound healing[J]. Cell Transplant. 2010;19:1635-1644. doi:10.3727/096368910X516637

24. Ackermann M, Pabst AM, Houdek JP, et al. Priming with proangiogenic growth factors and endothelial progenitor cells improves revascularization in linear diabetic wounds[J]. Int $J$ Mol Med. 2014;33 (4):833-839. doi:10.3892/ijmm.2014.1630

25. Critser PJ, Yoder MC. Endothelial colony-forming cell role in neoangiogenesis and tissue repair[J]. Curr Opin Organ Transplant. 2010;15 (1):68-72. doi:10.1097/MOT.0b013e32833454b5
26. Zhang M, Malik AB, Rehman J. Endothelial progenitor cells and vascular repair[J]. Curr Opin Hematol. 2014;21(3):224-228. doi:10.1097/MOH.0000000000000041

27. Zhang $\mathrm{J}$, Chen $\mathrm{C}$, $\mathrm{Hu} \mathrm{B}$, et al. Exosomes derived from human endothelial progenitor cells accelerate cutaneous wound healing by promoting angiogenesis through Erk1/2 signaling[J]. Int J Biol Sci. 2016;12(12):1472-1487. doi:10.7150/ijbs.15514

28. Gildea JJ, Carlson JM, Schoeffel CD, et al. Urinary exosome miRNome analysis and its applications to salt sensitivity of blood pressure[J]. Clin Biochem. 2013;46(12):1131-1134. doi:10.1016/j. clinbiochem.2013.05.052

29. Xin H, Li Y, Chopp M. Exosomes/miRNAs as mediating cell-based therapy of stroke[J]. Front Cell Neurosci. 2014;8:377. doi:10.3389/ fncel.2014.00377

30. Deiuliis JA. MicroRNAs as regulators of metabolic disease: pathophysiologic significance and emerging role as biomarkers and therapeutics[J]. Int J Obes (Lond). 2016;40(1):88-101. doi:10.1038/ ijo. 2015.170

31. Li Y, Song YH, Li F, Yang T, Lu YW, Geng YJ. MicroRNA-221 regulates high glucose-induced endothelial dysfunction[J]. Biochem Biophys Res Commun. 2009;381(1):81-83. doi:10.1016/j.bbrc.2009. 02.013

32. Liu HN, Li X, Wu N, et al. Serum microRNA-221 as a biomarker for diabetic retinopathy in patients associated with type 2 diabetes[J]. Int $J$ Ophthalmol. 2018;11(12):1889-1894. doi:10.18240/ ijo.2018.12.02

33. Presta LG, Chen H, O'Connor SJ, et al. Humanization of an anti-vascular endothelial growth factor monoclonal antibody for the therapy of solid tumors and other disorders[J]. Cancer Res. 1997;57 (20):4593-4599.

34. Yamagishi S. Role of advanced glycation end products (AGEs) and receptor for AGEs (RAGE) in vascular damage in diabetes[J]. Biochim Biophys Acta. 2011;46(4):217-224.

35. Fukami K, Yamagishi S, Okuda S. Role of AGEs $\square$ RAGE system in cardiovascular disease[J]. Curr Pharm Des. 2014;20(14):2395-2402. doi: $10.2174 / 13816128113199990475$

36. Jurikova M, Danihel L, Polak S, et al. Ki67, PCNA, and MCM proteins: markers of proliferation in the diagnosis of breast cancer[J]. Acta Histochem. 2016;118(5):544-552. doi:10.1016/j. acthis.2016.05.002

37. Ingham M, Schwartz GK. Cell-Cycle Therapeutics Come of Age[J]. $J$ Clin Oncol. 2017;35(25):2949-2959. doi:10.1200/JCO.2016. 69.0032

38. Huang JH, Yin XM, Xu Y, et al. Systemic administration of exosomes released from mesenchymal stromal cells attenuates apoptosis, inflammation, and promotes angiogenesis after spinal cord injury in rats[J]. J Neurotrauma. 2017;34(24):3388-3396. doi:10.1089/neu.20 17.5063

39. Perets N, Hertz S, London $M$, et al. Intranasal administration of exosomes derived from mesenchymal stem cells ameliorates autistic-like behaviors of BTBR mice[J]. Mol Autism. 2018;9:57. doi:10.1186/s13229-018-0240-6

Diabetes, Metabolic Syndrome and Obesity: Targets and Therapy

\section{Publish your work in this journal}

Diabetes, Metabolic Syndrome and Obesity: Targets and Therapy is an international, peer-reviewed open-access journal committed to the rapid publication of the latest laboratory and clinical findings in the fields of diabetes, metabolic syndrome and obesity research. Original research, review, case reports, hypothesis formation, expert opinion and commentaries are all considered for publication. The manuscript management system is completely online and includes a very quick and fair peer-review system, which is all easy to use. Visit http://www.dovepress.com/testimonials.php to read real quotes from published authors. 